Itinéraires Itinéraires

Littérature, textes, cultures

\title{
Paroles de banlieues : pour une redéfinition artistique des imaginaires
}

Words from the Suburbs: An Artistic Redefinition of the Imaginary

Ophélie Naessens

\section{OpenEdition}

Journals

Édition électronique

URL : http://journals.openedition.org/itineraires/3571

DOI : 10.4000/itineraires.3571

ISSN : 2427-920X

Éditeur

Pléiade

Référence électronique

Ophélie Naessens, «Paroles de banlieues : pour une redéfinition artistique des imaginaires », Itinéraires [En ligne], 2016-3 | 2017, mis en ligne le 15 juillet 2017, consulté le 20 avril 2019. URL : http:// journals.openedition.org/itineraires/3571 ; DOI : 10.4000/itineraires.3571

Ce document a été généré automatiquement le 20 avril 2019

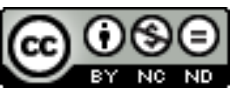

Itinéraires est mis à disposition selon les termes de la licence Creative Commons Attribution - Pas d'Utilisation Commerciale - Pas de Modification 4.0 International. 


\title{
Paroles de banlieues : pour une redéfinition artistique des imaginaires
}

\author{
Words from the Suburbs: An Artistic Redefinition of the Imaginary
}

\author{
Ophélie Naessens
}

1 En 1994 à Oakland, Californie, une émeute éclate à la fin du festival d'été dédié à la célébration de l'identité multiculturelle de la ville. Vers $18 \mathrm{~h} 30$, des centaines de jeunes gens descendent dans la rue pour affronter la police à l'aide de bouteilles et de pierres, des coups de feu sont signalés. Au bilan ; quinze personnes sont blessées et soixante-dixsept personnes, dont seulement six adultes, sont arrêtées. "Les gens couraient, brisaient des fenêtres, se battaient. C'était une émeute à grande échelle ${ }^{1}$. »

2 En 2005, la France est frappée par une vague d'émeutes qui fait suite à la mort de deux adolescents âgés de 15 et 17 ans, électrocutés lors de leur introduction dans l'enceinte d'un poste électrique tandis qu'ils tentaient d'échapper à un contrôle de police à Clichysous-Bois ${ }^{2}$. Très vite, les violences urbaines se propagent à travers les banlieues françaises, et donnent lieu à la déclaration de l'état d'urgence pour une durée de trois mois ${ }^{3}$. Au matin de la vingt-et-unième nuit, le bilan est de 9193 véhicules incendiés, 2921 interpellations, 56 policiers blessés et deux morts.

En province, c'est peut-être au Mirail à Toulouse que les affrontements ont été les plus importants. En dix jours, plus de deux cent cinquante voitures ont été brûlées et un jeune émeutier a eu la main arrachée par une grenade lacrymogène. [...] Le métro a cessé de desservir les quartiers sensibles après vingt heures [... $]^{4}$.

Depuis les années 1990, des artistes partent à la rencontre d'individus vivant dans des quartiers dits " sensibles », œuvrant à déconstruire l'imaginaire des banlieues produit par les médias et les acteurs politiques, ainsi qu'à en élaborer de nouvelles représentations. C'est ainsi dans ce contexte d'émeutes urbaines, distantes temporellement et spatialement, qu'ont choisi de travailler certains artistes, décidant de faire œuvre à partir de la parole des adolescents vivant sur place. Entre 1991 et 2001, l'artiste américaine Suzanne Lacy mène à Oakland un projet de grande ampleur en association avec TEAM 
(groupe réunissant adolescents, éducateurs, artistes et travailleurs des médias), mêlant installations, performances et activisme politique (The Oakland Project). Depuis 2009, les artistes suisses Frédéric Moser et Philippe Schwinger parcourent la France pour leur série filmique d'inspiration godardienne France, détours, en donnant la parole à des adolescents issus des différentes franges de la société. Pour le premier épisode intitulé «Devoir et déroute ", ils s'arrêtent au Mirail, à Toulouse, juste avant la mise en place du projet de rénovation du quartier. À partir de ces deux exemples, notre réflexion portera sur le rôle politique et social des arts visuels dans les représentations des banlieues. Nous aborderons la manière dont l'art est susceptible d'accueillir une nouvelle forme de contestation et de dissonance, à travers la découverte de discours inédits au sein de l'œuvre, ainsi qu'à travers la création d'espaces d'échanges et de dialogues autorisant le déplacement des discours et des images.

\section{Une situation conflictuelle}

4 Les situations géopolitiques et sociales dans lesquelles ces artistes interviennent sont habituellement considérées comme conflictuelles. À Oakland en Californie, la population des écoles publiques est constituée à $55 \%$ d'Afro-Américains et à $20 \%$ de LatinoAméricains, la jeunesse pâtit parallèlement de taux élevés de crimes violents, d'agressions sexuelles, de pauvreté et de décrochage scolaire (Lacy 2014). Le climat social et culturel d'Oakland dans le début des années 1990 est par ailleurs marqué par une séparation entre les quartiers défavorisés et les quartiers résidentiels aisés situés en périphérie de la ville, dans les « Hills ». Mais, au-delà d'une situation problématique réelle, les médias locaux et nationaux dépeignent la jeunesse afro-américaine et hispano-américaine unilatéralement sous ces aspects négatifs, ce qui entraîne des perceptions alarmistes, sinon erronées, visà-vis des quartiers pauvres et des jeunes de couleur. La perpétuation des représentations négatives de la jeunesse dans les médias donne lieu à un ancrage profond de ces clichés dans l'imaginaire des individus concernés. En effet, on peut supposer que les adolescents sont les spectateurs les plus assidus, mais aussi qu'ils ne possèdent pas forcément les outils et ressources pour critiquer ces stéréotypes. Comment alors pourraient-ils imaginer que leur trajectoire de vie puisse être différente de celle qu'on leur propose dans les médias, régie par la violence, l'absence d'instruction, le manque d'ambition professionnelle au profit d'activités délictueuses. Les émeutes de 1994 ont par exemple fait les gros titres des actualités du pays, et, en dépit du fait qu'une enquête diligentée après coup a permis de faire la lumière sur ces événements et notamment de clarifier le rôle de la police dans l'escalade de violence, le pays tout entier est resté marqué par ces images ${ }^{5}$.

5 En France, les instances politiques n'ont pas hésité à attiser la violence. En juin 2005, Nicolas Sarkozy, alors ministre de l'Intérieur, promet de « nettoyer les cités au kärcher ", et réitère ses provocations en octobre, interpellant les habitants du quartier du Val d'Argent à Argenteuil en leur lançant: «Vous en avez assez de cette bande de racaille, hein? On va vous en débarrasser. » Au Mirail à Toulouse, comme dans les autres zones sensibles touchées par les violences, les habitants sont les victimes du fossé qui se creuse entre eux et le reste de la France, supportant un sentiment d'injustice profond en termes d'emploi, de logement et d'éducation. De plus, leur situation géographique pèse sur leur intégration économique et sociale, et les stigmatise. Nombre de reportages ont été diffusés suite aux émeutes de 2005. Le traitement médiatique de celles-ci se base 
essentiellement sur un schéma qui se répète. Sur le plan des images, les visions apocalyptiques se succèdent; des rues sombres envahies par les flammes, jonchées de débris d'équipements et de voitures calcinées, assaillies par des jeunes cherchant à en découdre avec les forces de l'ordre. Le vocabulaire employé en voix off et apposé sur les images est guerrier, les reporters parlent d'" émeutiers ", de "cibles ", d'« armes », de «trophées ", etc. Quant aux jeunes interrogés par les journalistes, ils sont présentés cagoulés, dans des lieux obscurs, et il est parfois difficile de comprendre leurs propos. Encadrés par les questions ciblées des journalistes, ils tournent autour des mêmes thématiques: la violence, la drogue, les difficultés socio-économiques, etc. De cette manière, la télévision construit le spectacle de la réalité comme une fiction, comme une tragédie pourrait-on avancer, avec ses personnages et ses intrigues. Les médias élaborent ainsi une spectacularisation du réel qui donne lieu à une vision caricaturale de la banlieue. Davantage, on peut supposer que le traitement médiatique des événements en 2005 a participé, entre autres, à l'explosion de la situation ${ }^{6}$. À travers les reportages, les médias propagent des stéréotypes que les jeunes finissent par accepter, et qui peuvent devenir sources de fierté7.

6 Les tensions entre les différentes communautés: Afro-Américains / Blancs, jeunes / police, musulmans / chrétiens, habitants des zones urbaines sensibles / reste de la France, etc., ainsi que les problèmes inhérents à ces territoires, sont constamment soulignés par les médias, mais aussi par les instances politiques, à travers une surexposition des problématiques auxquelles elles sont confrontées. La «cité», le « ghetto », sollicitent un imaginaire peuplé de représentations négatives dont la pauvreté, l'insécurité, la drogue et la violence sont les caractères dominants. De par la généralisation de ces images et de ces discours, cette représentation partagée fait sens pour la majorité et stigmatise l'identité de ces communautés. Cette représentation, dont nous suggérons ici son lien avec l'idéologie de la classe dominante, est constamment nourrie par les grands médias nationaux et les instances politiques qui élaborent des visions de la réalité qui participent à créer en partie la réalité qu'ils s'attachent à décrire et à penser.

7 Dans les deux interventions qui nous intéressent, les artistes choisissent de travailler sur un réel discursif avec des communautés en proie à des conflits sociaux, mais aussi à cette intense période de questionnements qu'est l'adolescence, moment carrefour d'un devenir citoyen.

\section{L'espace de l'art : de l'émergence de nouveaux discours et de nouvelles images}

\section{The Roof Is on Fire}

8 Accompagnée d'Annice Jacoby et de Chris Jonhson, Suzanne Lacy organise l'événement The Roof Is on Fire ${ }^{8}$. Elle réunit deux cent vingt lycéens issus d'écoles publiques d'Oakland sur un parking aérien. Les adolescents, installés dans une centaine de voitures garées sur le toit, échangent sur différents sujets: violence, racisme, sexualité, drogue, avenir, famille, culture, éducation, etc. Cette performance a été réalisée sans scénario de dialogue établi à l'avance, devant un public d'un millier d'individus (habitants d'Oakland et médias locaux), invités à circuler de voiture en voiture pour écouter les conversations. The Roof Is On Fire fait suite à un travail engagé en amont pendant cinq mois entre l'artiste, des 
professeurs et des lycéens. Durant des ateliers préparatoires, les adolescents ont échangé sur les problèmes auxquels ils sont confrontés dans leur vie quotidienne, et ont également cherché à élaborer un message destiné aux pouvoirs publics et aux leaders politiques portant sur leur rôle dans le futur de la ville.

Pour Oakland Projects, Suzanne Lacy adopte une stratégie visant à agir contre les images fallacieuses exposées dans les médias et les séries télévisées. Sur le plan visuel, à première vue, les images de The Roof ressemblent à s'y méprendre à celles des nouvelles du soir et le choix d'un parking aérien n'est pas anodin; le lieu reprend les codes des images des quartiers déshérités qui prolifèrent dans les reportages et les séries télévisées. De plus, on retrouve ici une scène typique de la fiction que Lacy s'est employée à s'approprier et à modifier, celle de jeunes traînant dans des voitures sur des parkings obscurs, et se livrant à des activités forcément répréhensibles. Mais, contrairement au journal télévisé, l'histoire que raconte cette œuvre est différente, en premier lieu parce qu'ici, ce sont les jeunes qui se racontent et se représentent. Ainsi, l'artiste cherche à révéler la manière dont les jeunes se soucient de l'ouverture d'un dialogue et d'une sortie de la violence. Ce projet pose la question du possible développement d'un dialogue qui traverse les cultures et les communautés, sans pour autant sacrifier l'identité individuelle de chacun. L'enjeu est avant tout de produire une expérience esthétique susceptible de proposer une remise en cause de la perception de la différence, des identités fixes et des systèmes de connaissance. À travers le contexte ritualisé d'un événement artistique qui encadre des dialogues fondés sur une ouverture réciproque, chacun des acteurs est susceptible de s'exprimer au-delà de son rôle et de son identité prédéfinie. Les interlocuteurs sont invités à éprouver leur identité à travers leurs rencontres avec d'autres sujets, à ne plus se considérer uniquement à travers le prisme d'une perception subie par la société, mais en tant que sujets partageant des connaissances sur leurs opinions. L'échange dialogique proposé engage chacun à se voir à travers un autre point de vue, et ainsi à acquérir une conscience inédite de son discours. Cette conscience peut alors entraîner à son tour le développement d'une critique de soi à même d'engager les sujets à penser leurs opinions et identités non plus comme définitivement fixes, mais davantage comme contingentes et sujettes à des transformations créatives.

Dans The Roof, Suzanne Lacy choisit de donner la parole aux adolescents d'Oakland, leur donnant l'opportunité de parler en leur nom, et ce à grande échelle, et d'être écoutés9 Aussi, partant du principe que ces jeunes manquent d'outils critiques, Lacy et son équipe ont mis en place, parallèlement aux échanges menés, un programme d'éducation aux médias afin de donner aux participants les outils nécessaires pour comprendre et déconstruire les messages des médias de masse. Des débats qui éclosent dans The Roof Is on Fire, il ne s'agit pas de faire émerger des informations attendues, mais des paroles susceptibles de contester la réinterprétation généralement opérée par les médias, et ainsi de participer à l'élaboration d'un discours autre. En écoutant la parole des adolescents, le public de la performance découvre une jeunesse ouverte au dialogue, en proie à de nombreux questionnements mais aussi forte de revendications, contrastant fortement avec les préjugés éculés sur les jeunes des quartiers défavorisés. En outre, la série de dialogues improvisés livre un aperçu des préoccupations de ces jeunes, et plus précisément des difficultés auxquelles ceux-ci, lorsqu'ils appartiennent aux communautés afro et hispano-américaines, font face : stéréotypes des médias, " profiling racial », sousfinancement des écoles publiques, etc. Dans ce projet, l'artiste, accompagné par un dispositif pédagogique, œuvre à opérer une transition du stéréotype abstrait 
(" gangsta » / dealer, etc.) à la singularité, une transition qui ne peut s'opérer qu'à partir d'une redescription de leur identité par les individus concernés. En donnant à ces jeunes l'opportunité d'échanger entre eux, mais aussi de produire un message à destination d'une audience, The Roof Is on Fire leur offre la possibilité d'être des acteurs en décrivant eux-mêmes leurs situations et conditions d'existence. Ils reprennent le contrôle de leur image et de leur discours, construisent progressivement puis livrent au public une représentation d'eux-mêmes qu'ils ont choisie et pensée, laquelle transcende la dimension unilatérale promulguée par les médias «mainstream» et l'industrie du divertissement. Les discours qui émergent de la performance perturbent, d'une manière critique et créative, les discours ambiants. Les échanges retransmis, dont les contenus majeurs sont apparus durant la phase préparatoire du travail, ont produit un véritable débat. L'échange dialogique apparaît comme l'élément déclencheur d'un phénomène, celui de la transformation des perceptions convenues par l'expérience esthétique, en l'occurrence celle des adolescents noirs ou « hispanos » pensés comme des parias. En ce sens, ces événements artistiques s'appuient sur l'ambition de questionner, voire de contester les identités fixes et la perception de la différence. Cette conception renvoie à la pensée du philosophe Jean-Luc Nancy (1986), selon lequel nos identités sont toujours en négociation, inscrites dans un processus de formation et de reformation à travers nos rencontres avec d'autres. Le philosophe décrit un « être-hors-de-soi », à travers lequel les sujets pensent, agissent et parlent au-delà de leurs rôles a priori. Nancy oppose ainsi "l'être de la communication" au "sujet représentant» (1986: 62), soulignant la distinction à opérer entre une communication basée sur des sujets qui énoncent - et pardelà représentent - des opinions préexistantes, situation que nous retrouvons dans certains reportages par exemple, et une rencontre dialogique à travers laquelle la subjectivité est transformée par celle-ci, cas de figure ambitionné par les projets que nous évoquons ici.

\section{France, détours}

11 Pour la série de films France, détours ${ }^{10}$, Frédéric Moser et Philippe Schwinger s'inspirent d'une série de douze émissions réalisée par Jean-Luc Godard et Anne-Marie Miéville à partir de 1979 : France, Tour, Détour, deux enfants ${ }^{11}$, série elle-même librement inspirée du livre de lecture scolaire Le Tour de la France par deux enfants (Fouillée 1877). Pour le premier épisode Devoir et déroute présenté lors du festival de création contemporaine Le Printemps de septembre, Moser et Schwinger sont allés à la rencontre des adolescents de la cité du Mirail à Toulouse, un quartier réputé difficile, situé au cœur d'un ensemble d'immeubles conçu au début des années 1960 par l'architecte Georges Candilis.

Au commencement du tournage de Devoir et déroute, les artistes ont mené avec les adolescents des entretiens qui s'élaborent au fil des questions. La voix off du film précise le désir des deux artistes « de demander aux jeunes dont on nous dit qu'il faut avoir peur, s'ils n'ont pas besoin d'être protégés eux aussi. Non pas des cailloux lancés contre l'autorité, mais de la division du monde et des méthodes d'exclusion ${ }^{12}$ ». Les entretiens forment la composante majeure du film à travers des séquences dans lesquelles les jeunes habitants sont filmés répondant aux questions des artistes sur les lieux clés de la vie de la cité : la dalle, l'école, le stade, une association. Il ne s'agit pas de faire des portraits des jeunes à partir des entretiens, mais davantage de faire émerger un questionnement qui les conduise à penser leurs conditions d'existence et à se positionner en tant que sujet. 
13 Moser et Schwinger souhaitent réfléchir "en direct» avec les jeunes, posent des questions auxquelles ils ne possèdent pas de réponses, n'attendant pas d'informations précises. Leurs interrogations évitent les sujets prévisibles. Ils leur demandent par exemple : «Est-ce qu'il pourrait exister une matière qui fasse aussi un passage entre ce qu'on apprend, ce qu'on acquiert, et ce qu'on porte en soi ? », ou encore : «Est-ce que tu penses qu'il y a des intérêts derrière les images?» Les réponses sont parfois lentes, elliptiques, mais le cheminement se fait progressivement, patiemment. Les questions se succèdent dans l'écoute attentive du parcours de pensée de celui qu'on interroge. Elles ne sont jamais simplifiées, ils préfèrent répéter la question, et laisser le temps à la personne de répondre, plutôt que d'adapter le discours.

Dès la première scène du film, durant laquelle nous voyons à l'écran des images ralenties de la ville de Toulouse, puis du quartier du Mirail, surgit la voix d'un des artistes :

Ici, afin de sévir et de civiliser, on déclare le quartier : à risques. Mais comme par le passé, on prend soin de confondre danger et précarité. C'est une pratique de substitution utilisée par les illusionnistes: on met généralement une chose à la place d'une autre. Ici, on substitue à la violence faite à la multitude, en la parquant puis en la dispersant, une image de mixité comme réponse à l'insécurité.

Cette voix off intervient à différentes reprises dans le film, s'inscrivant principalement en contrepoint des images contemporaines du quartier. Elle leur apporte un autre éclairage, évoquant par exemple la construction et le découpage idéologiques des espaces urbains. Le texte engage en filigrane une réflexion quant à l'échec de cet ensemble d'habitation, dont l'inadéquation à la vie sociale ferait naître des tensions inhérentes à ce type de « cités » françaises. À certains moments, la voix off vient se superposer aux témoignages et renvoie alors ceux-ci à l'assujettissement idéologique dont ils se font parfois l'écho. À la fin de l'entretien avec Zora, la parole de l'adolescente se fait peu à peu recouvrir par une voix devenue over :

Zora par exemple, est une jeune fille comme une autre, cette expression qui ne dit à vrai dire rien, ici, prend sens, car cette jeune fille, manifestement, veut nous en donner la preuve, de sa ressemblance avec les filles de son âge [...].

Il n'y a pas ici de la part des artistes un projet documentariste à travers lequel ils chercheraient à faire émerger une version objective qui naîtrait de la confrontation de ces différentes "versions du monde", celle médiatique, politique, et celle vécue individuellement. C'est bien davantage la rencontre de postures et d'énoncés rivaux qui devient la condition nécessaire d'une didactique possible permettant aux artistes de mener une entreprise de « redescription » du monde.

17 Les rapports entretenus entre idéologie et parole des adolescents sont ici traités plastiquement à travers une redistribution des rôles de l'image et du discours. Les images de Devoir et déroute - succession de plans larges sur des espaces vides, désolés; barres d'immeuble, square abandonné, mais aussi des extraits d'archives télévisées présentant le projet idéaliste de l'architecte pour le Mirail, apparaissent comme support de l'idéologie, tandis que la parole des adolescents s'inscrit en contrepoint. Les entretiens filmés dévoilent quant à eux une forme de parole collective sur la cité, une réflexion en filigrane sur la constitution d'une identité et d'un imaginaire communs, qui infirme les représentations stéréotypées sur les grands ensembles. Des instants de vérité émergent des paroles, hors de tout contrôle et d'attente préformatée, bien que certains éléments lexicaux surgissent parfois subrepticement, se référant directement à cette idéologie, fragments intériorisés d'un discours assené. En effet, nous percevons parfois dans les 
discours des adolescents la présence d'un discours d'emprunt tenu par les médias à leur propos.

Néanmoins, l'évocation par ces jeunes habitants de leur vie quotidienne fait apparaître une réalité plus complexe de l'espace de la cité. Le film témoigne ainsi de la distorsion qui demeure entre discours médiatique et discours des citoyens. La coexistence de leurs paroles avec des énoncés rivaux au sein même des films fait émerger la conception d'une prise de parole comme lieu de l'expression d'un autre imaginaire possible, d'une contreidéologie. Frédéric Moser et Philippe Schwinger ouvrent ainsi un espace de réflexion qui interroge et déconstruit les présupposés et les évidences attachés à la construction de la réalité de ce territoire urbain. Ils développent leur objectif en ces termes :

$\mathrm{Si}$ nous parvenons à parler et à voir autrement des situations connues et reconnaissables de tous, les idéologies qui sous-tendent à la médiatisation de ces faits, lorsque ceux-ci sont traités dans les médias majoritaires, seront rendus perceptibles. (Moser et Schwinger 2009:6)

En donnant la parole à ces adolescents, les artistes invitent à la fabrication d'un « autre monde ", à un déplacement qui contribuerait à appréhender des réponses alternatives à l'idéologie dominante.

\section{De la circulation des paroles au-delà de l'œuvre}

\section{France, détours, retour(s)}

La réinscription et le réinvestissement des représentations de parole construites dans les films issus de la série France détours instaurent dans l'espace public une circulation des discours entre espaces-temps distincts. Cette circulation peut être conçue comme stratégie critique permettant d'envisager la création d'une nouvelle forme d'espace public de dialogue. Pour Moser et Schwinger, il est primordial que les films soient dans un premier temps projetés sur les lieux mêmes dans lesquels ils ont été tournés, par exemple au centre culturel Alban Minville ${ }^{13}$ pour le premier épisode. Ces propositions s'inscrivent dans une volonté des artistes de rencontrer à nouveau les acteurs ayant collaboré au projet, et les populations directement concernées par les discours et représentations qui sont en jeu dans les vidéos. Ils expliquent que la mise en place de sessions extraartistiques est une proposition pertinente pour leur travail (Moser et Schwinger 2011 : 15) :

En même temps que nous enclenchons un processus de travail, nous ouvrons un plateau de formulation, une tribune métaphorique à l'adresse de celles et ceux qui s'engagent dans des pratiques signifiantes, artistique, scientifique, sociale, mais aussi à l'adresse de celles et ceux qui se décrivent et se projettent dans notre contemporanéité en se sachant faisant partie d'un monde commun.

21 À travers des formes plus ou moins participatives, les deux artistes réinvestissent parfois l'espace public, dans le temps de l'exposition ou dans celui de la projection de films, à travers des débats, rencontres, workshops et autres rendez-vous, avec des participants aux films et/ou un public plus large. Lors d'une exposition à Bétonsalon en 2011, les artistes ont proposé trois rendez-vous "hors les murs " dont des rencontres visant à mettre en perspective les interrogations relatives au système éducatif et au rapport à l'autorité qui émergent de Ce trait, c'est ton parcours, le deuxième épisode de la série tourné sur un site expérimental dédié à l'accueil des collégiens exclus. Dans le cadre du colloque "Luttes universités contre la crise ${ }^{14}$ ", organisé par le groupe Edu-factory ${ }^{15}$ durant 
l'exposition, Frédéric Moser et Philippe Schwinger ont proposé à l'université Paris 8 (Saint-Denis) un workshop autour du projet France, détours. Ils le définissent ainsi (30) :

Notre objectif est de faire de l'éducation un débat. En partant de l'hypothèse que l'école est un lieu de diffusion des savoirs et des compétences susceptibles de donner à chacun les moyens d'augmenter son autonomie, sa puissance d'agir et de penser, comment parler de ce qui est en jeu au quotidien dans une leçon de français d'un collège de banlieue? L'école peut-elle être perméable à d'autres modes d'apprentissage que celui de la leçon ? Peut-on expérimenter de nouveaux modèles de transmission, sans devoir se situer entre l'un ou l'autre des pôles de l'antagonisme éducation/instruction, peu pertinent? Que faudrait-il pour que la pratique démocratique soit réellement mise en acte dans l'enseignement selon le projet «n'importe qui est capable d'apprendre n'importe quoi »?

À partir d'une projection des rushes de tournage, les deux artistes ont cherché à créer des moments d'échange et de débat avec les participants d'origines diverses présents au workshop. Ceux-ci étaient sollicités afin d'ouvrir des perspectives réflexives et critiques autour de la question du rapport à l'autorité, la sélection, l'exclusion dans le système éducatif, ainsi que sur le paradoxe qui lui est inhérent, d'être à la fois facteur de démocratisation et d'égalité des chances ainsi qu'un facteur de hiérarchisation qui perpétue l'ordre social établi.

\section{The Roof ... et après?}

Avec The Roof Is on Fire, Suzanne Lacy provoque une rencontre dans un espace d'entredeux, un «inbetween space», selon l'expression de Grant Kester (1998), désignant un « entre » des espaces différents (espace public / espace privé ; institution artistique / rue, etc.), un espace temporaire, qui exploite les interstices et les vacances des lieux publics. Cet espace n'est pas seulement physique, il se déploie également entre des sphères d'action différentes. En effet, comme le souligne Kester (2011: 205), ces projets «ne procèdent pas à travers une confrontation frontale directe avec l'État dans l'espace public de la rue, ni avec la complicité des canaux bureaucratiques existants, mais plutôt en travaillant dans un espace entre activisme manifeste et protocoles officiels ». Pour Lacy (1995 : 19), il s'agit de créer un art qui permettrait de « communiquer et d'interagir avec un public large et diversifié sur les questions directement liées à leur vie [...] sur la base de l'engagement ». Avec ce projet en particulier, l'artiste cherchait à entamer une forme de réhabilitation de ces jeunes, mais aussi à impacter plus largement les politiques sociales.

À travers l'intervention artistique, des dialogues constructifs ont pu voir le jour, et, davantage, conduire à des résultats et à des effets concrets. Pour The Roof, la présence des médias lors de la performance a entraîné des répercussions conséquentes à travers la retransmission des échanges. L'événement a en effet été diffusé par la chaîne d'actualité $\mathrm{CNN}$, la documentation vidéographique de la performance distribuée sous forme de documentaire à la télévision, et le projet relayé à travers des expositions, des conférences et des articles. Aussi, The Oakland Projects n'ont pas pris fin avec cette soirée on the Roof, mais se sont poursuivis pendant une dizaine d'années à travers un dispositif beaucoup plus large, dépassant le contexte artistique. La performance inaugurale a donné lieu à de 
nouvelles mises en scènes ${ }^{16}$, ainsi qu'à des programmes d'éducation artistique et aux médias à destination des adolescents, notamment grâce à l'implication d'enseignants sur place. Aussi, The Roof a donné lieu à de nombreuses initiatives, chevauchant les domaines de la politique publique, des relations entre jeunes et police, et de la réforme de l'éducation, apportant des pistes de réflexion face aux difficultés auxquelles font face les adolescents concernés.

D'aucuns pourront arguer que, sous l'angle de la transformation de la société, ces projets artistiques n'offrent qu'une modeste contribution effective. Néanmoins, ceux-ci s'affirment comme des expériences se nourrissant des images et des discours produits par la société dans laquelle ils font irruption, lui causant non pas un dommage, tel l'insecte parasite, mais introduisant dans l'espace et le débat publics de l'inattendu, du dissensus et du conflit, des éléments susceptibles d'éclairer sous un jour inédit les problématiques sociopolitiques actuelles.

\section{BIBLIOGRAPHIE}

Fouillée, Augustine, 1877, Le Tour de la France par deux enfants, Paris, Belin.

Kester, Grant H., 1998, « Socially Engaged Practice. Dialogical Aesthetics: A Critical Framework For Littoral Art », Variant, n 9, [En ligne], http://www.variant.org.uk/9texts/

KesterSupplement.html, consulté le 20 novembre 2014.

Kester, Grant H., 2011, The One and the Many: Contemporary Collaborative Art in a Global Context, Durham, Duke University Press.

Lacy, Suzanne (dir.), 1995, Mapping the Terrain, Seattle, Bay Press.

Lacy, Suzanne, 2014, « Nature, Culture, Public Space: Artist Statement », Finitude, [En ligne], https://www.cla.purdue.edu/waaw/Cohn/Artists/Lacystat.html, consulté le 8 janvier 2015.

Moser, Frédéric et Schwinger, Philippe, 2009, « Entretien par correspondance entre Jocelyn Wolff et Frédéric Moser et Philippe Schwinger, février 2009 », KOW ISSUE, n² 2, Paris, Galerie Jocelyn Wolff.

Moser, Frédéric et Schwinger, Philippe, 2011, « Note d'intention de l'exposition Ce dont on sera dans l'avenir capable ", Le journal de Bétonsalon, $\mathrm{n}^{\circ}$ 9, [En ligne], http://www.betonsalon.net/IMG/ pdf/bs9_betonsalon_finallight.pdf.

Nancy, Jean-Luc, 1986, La Communauté désœuvrée, Paris, Christian Bourgois.

\section{NOTES}

1. Le lieutenant Pete Peterson, interrogé par la station de radio KCBS, propos rapportés par l'AP, Associated Press, le 6 juin 1994, [En ligne], http://www.apnewsarchive.com/1994/Riot-atOakland-Festival/id-a12f3f7b641263c49d589b8cbed7b222 (traduction de l'auteur). 
2. Notons ici que le procès final de cette affaire a eu lieu en mars 2015 à Rennes, et a donné lieu à la relaxe des deux policiers concernés.

3. La mise en application de cette loi française datant du 3 avril 1955 est exceptionnelle, établie à l'origine pendant la guerre d'Algérie.

4. Journal de 13 heures, France 2, 17 novembre 2005, Archives de l'INA, https:// www.youtube.com/watch?v=gr5MM5XOHfo (consulté le 23 juin 2015).

5. On retrouve des précédents sur ce type de couverture médiatique, notamment lors des « Rodney King Riots » en 1991.

6. Voir à ce sujet le reportage diffusé par Canal Plus de Spécial Investigation «Émeutes en banlieue : la mécanique infernale ", le 31 octobre 2013.

7. Il a en effet été reconnu que les reportages télévisés ont contribué à une émulation négative entre les quartiers, participant à la mise en place d'une compétition visant le nombre de voitures brûlées.

8. Suzanne Lacy, The Roof Is on Fire, performance avec 220 adolescents, Annice Jacoby et Chris Johnson, Oakland, Californie, USA, 1994.

9. Il est intéressant de noter à ce propos que les participants, ayant pris en charge l'intégralité de la communication sur l'événement, ont choisi pour slogan de l'affiche : « Tais-toi et écoute».

10. Le second épisode a été tourné en 2011 en banlieue parisienne à Pierrefitte-sur-Seine avec des élèves en situation de décrochage scolaire (Ce trait-c'est ton parcours), tandis que le troisième épisode s'est déroulé à Marseille avec des jeunes issus de milieux aisés, avant leur entrée dans des formations et écoles supérieures (Ainsi arrivèrent-ils fébriles au seuil de la voie royale...).

11. Jean-Luc Godard et Anne-Marie Miéville, France, Tour, Détour, deux enfants, 1979, 12 émissions de 25 min., couleur, avec Camille Virolleaud, Arnaud Martin, Betty Berr, Albert Dray. Le projet documentaire de Jean-Luc Godard pour la télévision se fondait sur ses conversations filmées avec deux enfants, un garçon et une fille. Il les interrogeait dans leurs divers lieux de vie et de passage, sur des thèmes aussi variés que la réalité, la morale, les mots, la lumière, le pouvoir ou la géographie.

12. Les extraits cités ici sont tous issus de la transcription de l'auteur de la vidéo de Frédéric Moser et Philippe Schwinger, France, détours - Épisode 1: Devoir et déroute, 26 min. 30 sec., 2009.

13. Frédéric Moser et Philippe Schwinger, France, détours, épisode I. Devoir et déroute, vidéo couleur et son, 26 min. $36 \mathrm{sec}$., 2009. Présentation au centre culturel Albin Minville, Toulouse, Printemps de Septembre, 2009.

14. Dans sa présentation du colloque «Luttes universités contre la crise », le mouvement appelle les éventuels participants à "constituer un réseau puissant et transnational dans lequel développer des stratégies capables de contraster les attaques contre l'université et le welfare social à travers des conférences et des workshops, des tables rondes et des assemblées, nous proposons d'entamer une discussion autour de thèmes-clés tels que : la production autonome de savoirs, l'auto-formation, les luttes de réseau, l'organisation politique de l'université dans le commun. À l'intérieur des formes de production prédominantes - dans lesquelles sont intégrées les informations, les codes, les connaissances, les images et les affects - les subjectivités ont besoin d'une grande liberté ainsi que du libre accès aux réseaux de communication, aux banques de données, aux circuits culturels. L'alternative au dualisme public/privé - symétrique à l'alternative capitalisme / socialisme - est aujourd'hui la production du commun. » (Le journal de Bétonsalon, $\mathrm{n}^{\circ}$ 9, Paris, p. 29).

15. Edufactory est un regroupement (international) de chercheurs engagés qui travaillent notamment sur la question du partage des savoirs, la notion du commun, etc.

16. Citons entre autres Youth, Cops and Videotape (1995), série de dialogues entre jeunes et policiers, No Blood/No Foul (1995-1996), performance participative avec des adolescents et des policiers, Code 33 : Emergency Clear the Air! (1997-1999), dialogues improvisés sur un parking de 
centre commercial. Pour plus d'informations, se référer au site web de l'artiste: http:// www.suzannelacy.com/the-oakland-projects/.

\section{RÉSUMÉS}

Depuis les années 1990, des artistes partent à la rencontre des habitants de quartiers dits «sensibles", œuvrant à déconstruire notre imaginaire des banlieues. Dans un contexte d'émeutes urbaines, Suzanne Lacy (The Roof Is On Fire, 1994), Frédéric Moser et Philippe Schwinger (France, détours, 2009), décident de faire œuvre à partir de paroles d'adolescents. À travers l'étude de ces deux exemples, nous aborderons la manière dont l'art est susceptible d'accueillir une nouvelle forme de dissonance, par la découverte de discours inédits et le déplacement des représentations.

Since the 1990s, some artists go out to meet the inhabitants of so-called "sensitive" neighborhoods, working to deconstruct our imaginary of suburbs. In a context of urban riots, Suzanne Lacy (The Roof Is On Fire, 1994), Frédéric Moser and Philippe Schwinger (France, détours, 2009), decide to create from teenagers words. Through the study of these two examples, we will discuss how art could host a new form of dissonance, with the exploration of novel speeches and the displacement of representations.

INDEX

Keywords : suburb, testimony, video, performance, dialogical art

Mots-clés : banlieue, témoignage, vidéo, performance, art dialogique

\section{AUTEUR}

\section{OPHÉLIE NAESSENS}

Université de Lorraine, Crem (EA 3476) 